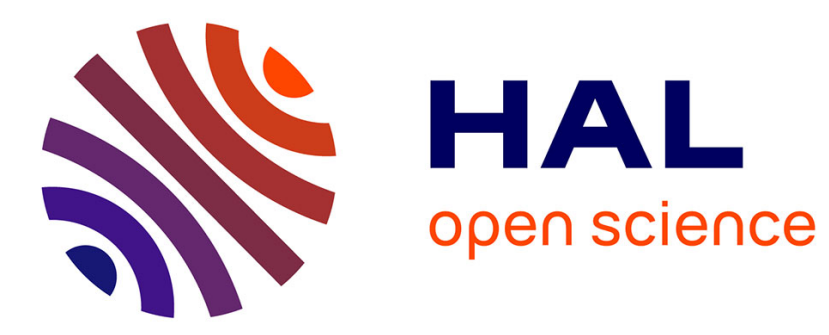

\title{
Le maire et ses partenaires : du schéma centre-périphérie à la gouvernance multi-niveaux
}

Gilles Pinson

\section{To cite this version:}

Gilles Pinson. Le maire et ses partenaires: du schéma centre-périphérie à la gouvernance multiniveaux. Pouvoirs - Revue française d'études constitutionnelles et politiques, 2014, Le maire, 148, pp.95-111. 10.3917/pouv.148.0095 . halshs-00965084

\section{HAL Id: halshs-00965084 \\ https://shs.hal.science/halshs-00965084}

Submitted on 4 Jan 2022

HAL is a multi-disciplinary open access archive for the deposit and dissemination of scientific research documents, whether they are published or not. The documents may come from teaching and research institutions in France or abroad, or from public or private research centers.
L'archive ouverte pluridisciplinaire HAL, est destinée au dépôt et à la diffusion de documents scientifiques de niveau recherche, publiés ou non, émanant des établissements d'enseignement et de recherche français ou étrangers, des laboratoires publics ou privés. 


\section{LE MAIRE ET SES PARTENAIRES: DU SCHÉMA CENTRE-PÉRIPHÉRIE A LA GOUVERNANCE} MULTI-NIVEAUX

i d'un point de vue juridique la catégorie de «maire» a encore un sens, d'un point de vue analytique elle n'en a plus guère. Malheureusement, la science politique française n'en a pas encore pris la mesure. La discipline a eu beau vouloir s'affranchir du droit et abandonner des lectures par trop formalistes et institutionnalo-centrées des phénomènes, des organisations et des rôles politiques, elle en reste malheureusement encore prisonnière. J'en veux pour preuve le maintien de la funeste notion générique de «local» qui, si elle a eu son utilité dans les décennies 1960, 1970 et 1980 pour éveiller l'attention sur les fonctionnements concrets des espaces politiques subnationaux jusqu'alors tenus en grand mépris par la discipline, tend aujourd'hui à masquer le fait qu'il n'y a plus grand-chose aujourd'hui de commun entre le maire d'une commune rurale et le président d'une communauté urbaine.

La science politique nord-américaine - sans doute plus attentive à ce qui s'écrit dans les autres disciplines qui s'intéressent à l'urbain - a depuis longtemps pris acte du fait que la différenciation des structures sociales, des rôles économiques et des modalités de la mobilisation politiques propres à chaque configuration territoriale - métropole, banlieues résidentielles, petites villes, espaces ruraux - exigeait des modes d'appréhension spécifiques et le recours à un vocabulaire permettant de «ventiler» le local, le subnational en une variété d'objets politiques: l'urbain, le suburbain, le rural, etc. Cette sensibilité aux logiques de 
différenciation a produit un groupe spécifique au sein de l'Association américaine de science politique (APSA).

Rien de tel en France où l'urbain n'est bien souvent pas pris au sérieux, ou laissé aux urbanistes. On peut sans doute y voir le produit d'une logique d'imprégnation des milieux scientifiques par l'humeur nationale jacobine et par un modèle de développement national qui a associé Paris et les campagnes contre les grandes villes de province incarnant pêle-mêle les corporations, la société civile, les bourgeoisies, le marché ${ }^{1}$. Autant d'éléments que le modèle de développement de l'État - qu'il soit monarchique, impérial, républicain ou providence - a toujours voulu tenir à distance. S'il y a une idéologie anti-urbaine en France, c'est moins celle qui vise Paris ${ }^{2}$ que celle qui stigmatise les cloaques de médiocrité que seraient les villes de province. Il n'y a guère que l'École des Annales qui en France ait voulu conjurer ce tropisme.

En science politique, la tentative des tenants du chantier de la gouvernance urbaine ${ }^{3}$ qui portait en filigrane cette volonté de donner plus d'épaisseur à une approche politologique des villes comme espaces spécifiques de mobilisation politiques et d'action publique s'est heurtée à une double levée de boucliers, venant d'horizons scientifiques a priori diamétralement opposés mais se rejoignant au final dans une posture commune. La première fut celle des tenants d'une approche en termes d'administration locale, souvent proches du droit et de la science administrative et refusant la perspective d'un décentrement dans l'analyse des institutions et des régulations politiques, et plus globalement attachés à une vision dans laquelle les collectivités sont avant tout des découpages administratifs d'un État central demeurant un acteur ultra-dominant ${ }^{4}$. La seconde, plus vivace aujourd'hui, est le fait des tenants d'une sociologie bourdieusienne des champs politiques locaux inaugurée par le regretté Jacques Lagroye ${ }^{5}$. Cette approche défend également l'idée d'une

1. Aux États-Unis, si la classe politique et les groupes sociaux dominants ont souvent développé un tropisme pastoraliste et anti-urbain, cela n’a pas empêché la science politique de développer un intérêt pour le rôle des villes dans le processus de développement du système politique états-unien. Cf. Richardson Dilworth (dir.), The City in the American Development, New York (N. Y.), Routledge, 2009.

2. Un autre tropisme français, qui consiste à limiter l'urbain à Paris, s'exprime bien dans un ouvrage qui est censé traiter de la haine de la grande ville. Cf. Bernard Marchand, Les Ennemis de Paris. La haine de la grande ville des Lumières à nos jours, Rennes, Presses universitaires de Rennes, 2009.

3. Patrick Le Galès, «Du gouvernement des villes à la gouvernance urbaine », Revue française de science politique, vol. 45, $\mathrm{n}^{\circ}$ 1, 1995, p. 57-95.

4. Albert Mabileau, Le Système local en France, Paris, Montchrestien, 1991.

5. Parmi de nombreuses références, on citera Philippe Garraud, «Le métier d'élu local: les contraintes d'un rôle», in Joseph Fontaine et Christian Le Bart (dir.), Le Métier d'élu 
constante centralité des régulations politiques et institutionnelles mais surtout la thèse de la constitution des espaces politiques en des champs relativement clos au sein desquels les comportements des acteurs sont gouvernés par des logiques de concurrence et des prescriptions de rôle propres à chacun des champs. Si cette approche est fort utile et a immensément contribué à renouveler les approches du local, elle a pour principal défaut son caractère «campo-centré» et sa difficulté à voir dans quelle mesure les champs politiques locaux sont travaillés par des phénomènes extérieurs comme le changement social (négligé au nom de la sensibilité à la reproduction), les transformations du capitalisme (non prises en compte pour ne pas s'exposer au procès en fonctionnalisme), ou encore les transformations des espaces et des technologies (définitivement hors radar). Ces deux approches, à partir de postures diamétralement opposées, se complètent pour faire perdurer des visions génériques et universalisantes des rôles et des espaces politiques «locaux» ou «subnationaux» et des visions indifférenciées des «maires».

Or, lorsque l'on entend s'intéresser aux rapports qu'entretiennent les maires avec les autres échelons politiques, la caducité de cette approche générique saute aux yeux. En effet, comment peut-on sérieusement formuler l'hypothèse selon laquelle les rapports qu'un maire entretient avec le président du département, celui de la région, avec les services centraux et déconcentrés de l'État, mais aussi avec les nouveaux échelons que constituent l'intercommunalité et les institutions européennes, sont les mêmes selon que l'on parle du maire d'une commune rurale ou du maire-président d'une prospère communauté urbaine ? Il est deux autres conceptions enracinées dans la lecture classique du local dont il importe également de se départir. La première tend à considérer que les rapports avec l'État sont les plus déterminants pour comprendre le pouvoir et la capacité d'action d'un maire. En nous aidant des perspectives offertes par les travaux sur la gouvernance multi-niveaux, nous tenterons de démontrer que, si ces relations gardent une place éminente, il convient de ne pas les surestimer. La seconde consiste à penser que le pouvoir et la capacité d'action d'un maire proviennent essentiellement de sa faculté à capter des ressources à d'autres échelles via notamment le cumul des mandats. Je souhaiterais montrer ici que, dans un contexte de recomposition des politiques territoriales de l'État et de néolibéralisation, c'est

local, Paris, L'Harmattan, 1994, p. 29-54 ; Rémi Le Saout, «L'intercommunalité, un pouvoir inachevé», Revue française de science politique, vol. 50, n³ 3, 2000, p. 439-461; et Michel Koebel, Le Pouvoir local, ou la Démocratie improbable, Bellecombe-en-Bauges, Le Croquant, 2006. 
davantage de la capacité à mobiliser des ressources locales que proviennent la capacité d'action du maire et, au final, sa capacité à influer sur d'autres niveaux. C'est ainsi à un processus cumulatif que l'on assiste: les leaders politiques de territoires riches à la fois en ressources fiscales, d'expertise, mais aussi riches en groupes sociaux et professionnels et en groupes d'intérêts porteurs de ressources pour l'action publique, auront davantage de chances de constituer une capacité d'action; cette capacité d'action constituera un avantage pour s'imposer dans les relations intergouvernementales; elle sera portée à leur crédit par d'autres niveaux, alors plus disposés à leur allouer des ressources sous formes de financements, de labels et autres récompenses.

Dans la première partie de cet article, nous nous attacherons à décrire la situation empirique, révolue selon nous, dans laquelle s'enracinent les visions «classiques» du local et des maires. Pour cette description, des typologies canoniques des systèmes de gouvernement local élaborées dans le cadre de recherches comparatives seront sollicitées. Dans ces modèles, la relation principale dans laquelle sont impliqués les maires est une relation verticale avec l'État central. On y parle d'ailleurs de rapports centre-périphérie, ce qui suggère le caractère strictement bilatéral des systèmes de relations dans lesquels sont enserrés les maires. Si cette relation bilatérale est largement asymétrique, au profit de l'État central et au détriment des maires, elle a néanmoins deux vertus. D’abord elle leur réserve une capacité d'accès à l'État entendu comme ensemble d'institutions politique et administratives situés au centre, mais aussi comme réseau d'administrations déconcentrées. Ensuite, elle a un effet nivelant: elle offre potentiellement à un grand nombre de maires une capacité d'influence, quelle que soit la taille de la commune.

Dans la seconde partie sera montré que ce système de rapports centrepériphérie bilatéraux a explosé, laissant place à une dynamique de multilatéralisation des relations dans lesquelles sont impliqués les maires. La littérature sur laquelle nous nous appuierons préfère d'ailleurs parler de «relations intergouvernementales», ou encore de "gouvernance multi-niveaux », signes que l'État n'a plus le monopole des relations avec les communes et les maires. Les «partenaires» des maires ne se résument plus aux services de l'État, ils intègrent également les structures intercommunales, les départements, les régions, mais aussi l'Union européenne et, surtout, les communes d'autres pays organisées souvent en réseaux et associations. Au-delà, on peut même considérer que, s'agissant des grandes villes, les partenariats se sont développés avec les groupes sociaux, les acteurs économiques et, plus généralement, tous 
les segments des sociétés locales porteurs de ressources pour l'action publique. Ce système multilatéralisé, s’il libère les maires de leur têteà-tête avec l'État, est porteur de logiques très fortes de différenciation, voire d'inégalités croissantes entre les communes. Dans ce système, les maires-présidents qui dirigent de puissantes institutions métropolitaines sont davantage capables d'imposer leurs agendas que les maires de communes rurales. Et dans cette configuration, le salut ne peut plus guère venir des facultés d'accéder au centre par le cumul des mandats ou les relations quotidiennes avec les services de l'État.

\section{LE SYSTÈME NAPOLÉONIEN : \\ DES MAIRES DOMINÉS MAIS ÉGAUX ET INFLUENTS}

Il fut un temps où les conditions de fonctionnement de la République mais aussi les formes du capitalisme français et le développement du welfare state assuraient une relative égalité entre maires dans leur capacité d'influencer d'autres niveaux. L'extrême fragmentation de la carte communale, l'uniformité du statut des communes conformément au modèle napoléonien, la pratique du cumul des mandats, la présence des maires ruraux au sein de puissants conseils généraux mais aussi au Sénat, le poids de la ruralité dans l'imaginaire national, étaient autant d'éléments qui pouvaient donner du poids aux maires, y compris ceux présidant aux destinées de petites communes rurales. Les conditions de consolidation de la III République avaient fait des maires ruraux et des petites patries qu'ils gouvernaient le ciment du régime à une époque où l'administration centrale était soupçonnée de sympathie impériale ${ }^{6}$. Le poids de l'Association des maires de France témoignait de ce pouvoir potentiel des maires, y compris des plus «petits» parmi eux ${ }^{7}$. L'hostilité de la force politique structurante de la III ${ }^{e}$ République, les radicaux, à la ville et à l'industrialisation participait à contenir le rôle des maires urbains. Par ailleurs, un mode de développement économique fondé sur l'agriculture et la petite industrie, en vertu du fameux "référentiel de maintenance ${ }^{8}$ »,

6. Bruno Dumons et Gilles Pollet, «Espaces politiques et gouvernements municipaux dans la France de la III ${ }^{e}$ République. Éclairage sur la sociogenèse de l'État contemporain », Politix, vol. 53, n 14, 2001, p. 15-32.

7. Patrick Le Lidec, Les Maires dans la République. L'Association des maires de France, élément constitutif des régimes politiques français depuis 1907, thèse de science politique, Université de Paris 1-Panthéon-Sorbonne, 2001.

8. Pierre Muller, «La politique agricole française: l'État et les organisations professionnelles», Économie rurale, vol. 255, n 1 , 2000, p. 33-39. 
assurait à chaque territoire un rôle productif et, par voie de conséquence, un minimum de ressources à un vaste nombre de gouvernements locaux.

La réactivation du colbertisme dans l'après-guerre et le développement du welfare state consolidèrent encore un peu plus ces conditions d'une distribution relativement équitable des capacités d'accès des maires aux instances supérieures. La redistribution de l'appareil productif par les grâces du «keynésianisme spatial ${ }^{9}$ » et le développement d'un réseau dense d'équipements du welfare à travers l'ensemble du territoire permirent de pérenniser le réseau des villes moyennes et d'irriguer ainsi les territoires ruraux. Cette donne rendit possible la préservation du pouvoir des notables locaux, comme en témoigne la survie des coalitions de troisième force à l'échelle des conseils municipaux, composées des forces politiques représentatives de la France rurale et des petites villes, jusque très tard sous la $\mathrm{V}^{\mathrm{e}}$ République alors même que le nouveau régime les avait marginalisées au plan national.

L'importance des communes et des maires dans l'organisation économique, sociale et spatiale du pays mais aussi dans son développement et son fonctionnement politique a été relevée par les études comparatives sur le rôle et le poids des gouvernements locaux en Europe occidentale. Ces travaux, notamment ceux de Michael Goldsmith et Edward Page ${ }^{10}$ et de Joachim Hesse et Lawrence Sharpe ${ }^{11}$, sont assez anciens mais ils ont l'avantage de bien rendre compte de la situation de départ et, en particulier, de la nature des relations intergouvernementales dans lesquelles étaient pris les maires jusque très récemment. Ces travaux ont en commun d'intégrer le cas français dans une catégorie - celle des systèmes «méridionaux» chez Goldsmith et Page ou «franco» chez Hesse et Sharpe - qui les distingue des autres systèmes - «septentrionaux» chez les premiers et «anglo» et «nord et mitteleuropéens » chez les seconds - en fonction de critères qui varient d'une typologie à l'autre: le nombre et la nature des compétences exercées, le degré de discrétion ou d'autonomie dans l'exercice des compétences, le statut constitutionnel, le contrôle exercé par les échelons supérieurs (notamment l'État) et, enfin et surtout, les capacités d'accès au centre.

9. Neil Brenner, New State Spaces: Urban Governance and the Rescaling of Statehood, Oxford, Oxford University Press, 2004.

10. Edward Page, Michael Goldsmith (dir.), Central and Local Government Relations: A Comparative Analysis of West European Unitary States, Londres, Sage, 1987.

11. Joachim Hesse et Lawrence Sharpe (dir.), Local Government and Urban Affairs in International Perspective, Baden-Baden, Nomos, 1991. 
Ainsi, dans le type «méridional», «franco», parfois aussi appelé «napoléonien", les enjeux de contrôle du territoire et de surveillance des périphéries étaient classiquement centraux du fait du poids de l'héritage féodal et des situations de fragmentation et d'affaiblissement du pouvoir central qui lui étaient associées. Dans ce modèle, l'élu local est à la fois un frondeur à surveiller et potentiellement un relais du pouvoir central. Les deux positions lui donnent en tout cas un rôle éminent. Cette double position se traduit très tôt par un mélange étrange d'intrusion du centre dans les affaires locales (en la personne du préfet) et de reconnaissance des libertés locales, attestée par la reconnaissance constitutionnelle du rôle des gouvernements locaux, l'existence de conseils élus et le poids politiques des élus locaux. Toutes choses qui contrastent avec la plus grande autonomie dont jouissent les gouvernements locaux des pays du nord de l'Europe mais qui est étrangement associée à un caractère très dominé, subalterne, du personnel politique local. Ainsi le caractère historiquement tendu des rapports centre-périphérie a-t-il généré un système politique local et des élus locaux dont on attend une capacité à défendre la community locale lorsque l'on attend de leurs homologues du Nord une capacité à gérer avec efficiency les affaires locales et, de plus en plus, les services du welfare ${ }^{12}$.

D'où l'importance dans les systèmes de gouvernement local et de rapports centre-périphérie méridionaux des enjeux et des possibilités d'accès du personnel politique local à l'État et ses différentes expressions. Cet accès est assuré par la pratique du cumul des mandats - peu ou pas présente dans les pays du Nord - qui non seulement permet aux élus de peser dans les décisions prises au Parlement mais leur donne également du poids dans leurs relations avec les représentants locaux de l'État. Il est aussi garanti par un système de production des politiques publiques que l'on dit «fusionné». Au sein de ce système, il n'existe pas de répartition des compétences et de division des tâches - entre conception et mise en ouvre, par exemple - très claires entre l'État et les collectivités territoriales, à l'inverse du système dual ${ }^{13}$ typique du Royaume-Uni. Dès lors, l'État est doté de services déconcentrés répartis sur le territoire selon un maillage très fin et participant en collaboration avec les collectivités territoriales à la conception et à la mise en œuvre des politiques

12. Brian Smith, Decentralization: The Territorial Dimension of the State, Londres, Allen \& Unwin, 1985.

13. James Bullpit, Territory and Power in the United Kingdom, Manchester, Manchester University Press, 1983. 
publiques, et ce dans une très grande variété de ces dernières. Il faut préciser que ce dispositif n'a pas attendu la décentralisation pour voir le jour, même si cette dernière lui a donné encore plus d'importance avec la pratique, en particulier, de la contractualisation ${ }^{14}$.

Dans cette configuration, les principales relations dans lesquelles sont impliqués les maires - ou celles qu'ils jugent les plus stratégiques - sont les relations verticales les mettant en lien avec les représentants locaux et centraux de l'État, et dans une moindre mesure avec les élus et les services du conseil général. Les relations horizontales, celles associant les élus d'un même niveau et, en premier lieu, les maires, sont pour leur part très secondaires, voire marquées par l'évitement. C'est ce schéma relationnel que les sociologues des organisations ont qualifié de « régulation croisée ${ }^{15}$ ». L'échange se fait essentiellement entre deux types d'ac102 teurs interdépendants: les élus, notamment les maires, détenteurs de la légitimité élective et capables de réunir les conditions de l'acception des politiques de l'État par les sociétés locales; les techniciens de l'État détenteurs, eux, d'une légitimité technique et des ressources financières et d'expertise. Les maires ont davantage intérêt à soigner ces relations avec l'État, seules à même de fournir des ressources pour mettre en œuvre leur agenda. Les autres maires sont des rivaux dans ce jeu de captation des ressources de l'État. Pour sa part, l'État, toujours soucieux de maîtriser les capacités d'auto-organisation du local, entretient plutôt la situation au nom du principe «diviser pour mieux régner».

Ce système de partenariat essentiellement vertical explique largement pourquoi la France n'est pas parvenue jusque récemment à « rationaliser» son système communal et à réduire le nombre de communes, contrairement à ses voisins à l'est et au nord ${ }^{16}$. Non seulement parce que la présence du lobby des élus municipaux au Parlement a souvent fait échec à de telles réformes, mais aussi parce que la régulation croisée - dont l'État a toujours été protagoniste et complice -, associée à l'abondance des ressources, a entretenu des jeux compétitifs entre les communes. On verra dans la seconde partie que c'est seulement avec la raréfaction des

14. Jean-Pierre Gaudin, Gouverner par contrat: l'action publique en question, Paris, Presses de Sciences Po, 1999.

15. Michel Crozier, Jean-Claude Thoenig, «La régulation des systèmes organisés complexes: le cas du système de décision politico-administratif local en France ", Revue française de sociologie, vol. 16, 1, 1975, p. 3-32; Pierre Grémion, Le Pouvoir périphérique, Paris, Seuil, 1976.

16. Rappelons que la Suède a divisé depuis l'après-guerre le nombre de ses communes par dix, l'Angleterre par quatre le nombre de ses districts, et que l'Allemagne était passée de vingtcinq mille communes à huit mille cinq cents à la veille de la réunification. 
ressources consécutive à l'entrée dans une crise durable, avec le déclin tendanciel des politiques territoriales de l'État et de la présence de ce dernier au niveau déconcentré, et avec l'émergence du spectre d'une compétition territoriale à une plus large échelle (la compétition entre les régions et les métropoles) que des jeux coopératifs horizontaux ont pu se mettre en place sous la forme du pis-aller de la coopération intercommunale.

Il y a sans doute un autre aspect du modèle français d'organisation territoriale - et des voies d'accès du local au centre et du modèle relationnel qui lui sont associés - qui explique son «succès» auprès des maires et sa longévité. C'est son caractère nivelant, voire égalitaire. Dans ce système, ce qui donne à un maire un pouvoir d'influence sur les autres niveaux, et principalement sur les décisions étatiques, ce ne sont ni la taille du territoire qu'il représente ni même sa richesse, ce sont essentiellement des ressources politiques et institutionnelles: le contrôle de mandat et l'accès à des circuits d'influence. Dès lors, un maire d'une commune rurale pauvre mais cumulard et bien introduit peut peser $\mathrm{du}$ même poids que le maire d'une grande ville. Ainsi, les ressources politiques accumulées par l'homme politique (via ses capacités d'accès) comptent pour une grande part et peuvent très largement compenser la faiblesse des ressources propres du territoire. D'une certaine manière, les modèles méridionaux de rapports centre-périphérie assurent des formes de redistribution ou de saupoudrage du poids politique et, au final, des ressources publiques entre les territoires. C'est cette dimension que la période récente a largement remise en cause.

\section{LES MAIRES DANS LA GOUVERNANCE MULTI-NIVEAUX}

La réalité empirique sur laquelle étaient fondés les modèles classiques d'analyse des relations centre-périphérie a vécu. Si en France, comme indiqué en introduction, beaucoup d'observateurs peinent à le reconnaître, certains des créateurs des typologies canoniques n'ont pas d'états d'âme à le faire. Ainsi, dans une sorte de deuxième édition de leur ouvrage devenu classique, Michael Goldsmith et Edward Page abandonnent-ils l'expression «rapports centre-périphérie » pour lui préférer celle de «relations intergouvernementales » pour insister sur la fin d'un système dans lequel les maires étaient cantonnés à un tête-à-tête exclusif avec l'État. «Ce qui, vingt ans auparavant, apparaissait comme une affaire [les relations entre niveaux de gouvernement] limitée à des activités domestiques au sein des États-nations a aujourd'hui une dimension multi-niveaux, impliquant un nombre croissant de tiers à l'intérieur de chaque pays et 
par-delà les frontières, sans oublier l'influence de l'Union européenne ${ }^{17}$.» L'émergence de ce que les spécialistes de politique européenne ont appelé la «gouvernance multi-niveaux ${ }^{18}$ » a ainsi fait exploser le système relationnel classique de la régulation croisée décrit dans la première partie. Dans cette seconde partie, nous tenterons d'abord d'expliquer les différentes causes à l'origine de ce bouleversement, pour revenir ensuite sur les nouvelles formes de relations et de partenariats dans lesquelles sont impliqués les maires. Pour finir, nous insisterons sur le caractère de plus en plus différencié des types de relations intergouvernementales au sein desquels les maires sont engagés.

On peut distinguer trois ordres de processus qui ont bouleversé les systèmes de relations intergouvernementales: la transformation des rapports du capitalisme aux territoires et la métropolisation; les transformations des rapports de l'État au(x) territoire(s); la construction européenne. Ces différents facteurs ont profondément affecté la capacité qu'ont les maires d'accéder à d'autres échelles et à influer les processus de fabrication de l'action publique. Nous faisons l'hypothèse qu'ils ont eu également pour effet de rompre la logique égalitariste qui mettait tous les maires à peu près au même niveau dans la situation précédente. De surcroît, une autre hypothèse peut être avancée: cette capacité est moins garantie par l'accumulation de mandats électifs que par la position des maires au sein de territoires pourvoyeurs de ressources pour l'action publique.

Les transformations des systèmes productifs, des rapports du capitalisme aux territoires et la métropolisation constituent un premier ordre de facteurs qui ont précipité le changement. La sociologie et la géographie économiques ${ }^{19}$ ont abondamment démontré que dans le capitalisme mondialisé et post-fordiste les territoires qui «gagnaient» en Occident étaient non plus ceux qui étaient en mesure de fournir des facteurs de production génériques (main-d'œuvre, matières premières) en masse et donc d'accueillir les opérations routinisées de la production en série, mais ceux qui, à l'inverse, pouvaient offrir un cadre pour l'épanouissement des activités non routinières et peu codifiées (innovation, recherche, services de pointe aux entreprises, etc.) qui sont génératrices

17. Michael Goldsmith, Edward Page (dir.), Changing Government Relations in Europe: From Localism to Intergovernmentalism, Londres, Routledge, 2010, p. 1 (nous traduisons).

18. Liesbet Hooghe, Gary Marks, Multi-Level Governance and European Integration, Boulder (Colo.), Rowman \& Littlefield, 2001.

19. Cf. notamment Pierre Veltz, Mondialisation, villes et territoires. L'économie d'archipel, Paris, PUf, 1996; Michael Storper, Keys to the City: How Economics, Institutions, Social Interactions, and Politics Shape Development, Princeton (N. J.), Princeton University Press, 2013. 
de valeur ajoutée. Ces territoires, ce sont des écosystèmes territoriaux très spécifiques (espaces technopolitains, districts industriels) et, de plus en plus, les métropoles. On a là une clé du processus tant commenté aujourd'hui de la «métropolisation» dont on n'a pas encore bien saisi les implications en matière de représentation politique. Le processus de métropolisation est défini par Ludovic Halbert comme une dynamique de «concentration des hommes et des activités dans les principales agglomérations d'un système urbain donné ${ }^{20}$ ». La métropolisation opère diamétralement à rebours du processus de redistribution territoriale et de consolidation de la trame des villes moyennes que le développement de l'État-providence avait activé et que nous avons décrit en première partie. Elle prive de plus en plus de territoires de toute fonction économique au profit d'une poignée de grandes agglomérations. Les prive-t-elle également de leur poids politique? Il est tentant de le croire lorsque l'on observe à quel point les dernières décisions en matière de grands équipements universitaires (plan Campus, programme des investissements d'avenir, etc.) ont concentré l'effort de l'État sur quelques métropoles sans que les élus des autres territoires semblent en mesure de contrarier cette logique de concentration. La métropolisation paraît ainsi opérer un grand tri non seulement économique mais aussi politique entre territoires et détermine selon nous la capacité des maires à s'imposer dans leurs relations avec les autres niveaux.

Les transformations des rapports de l'État au(x) territoire(s) constituent une seconde source de l'évolution des rapports centre-périphérie. On l'a dit, ce qui a historiquement motivé la structuration du modèle méridional ou napoléonien de rapports centre-périphérie, c'était la volonté de la part de l'État d'assurer la cohésion d'un ensemble national fragmenté et agité par les tentations frondeuses des périphéries. C'était aussi la nécessité de défendre le territoire contre des ennemis situés au-delà des frontières. Or, en suivant encore Goldsmith et Page, on peut considérer que l'une et l'autre de ces motivations sont beaucoup moins d'actualité, au moins en ce qui concerne le cas de la France. Ainsi, on peut penser que, si les pays du modèle napoléonien se sont pour la plupart décentralisés, c'est que le modèle a parachevé sa mission d'unification et que la menace qui gronde aux frontières n'est plus qu'économique. Ces évolutions sont sans doute heureuses à certains égards, mais elles privent bon nombre de territoires de leur intérêt stratégique pour l'État et font perdre à leurs élus une part importante de leur poids dans les relations

20. Ludovic Halbert, L'Avantage métropolitain, Paris, PUF, 2009, p. 6. 
intergouvernementales. Les transformations des rapports de l'État au(x) territoire(s) ont récemment trouvé une magnifique illustration à travers la Réforme de l'administration territoriale de l'État (REATE) ${ }^{21}$. Conduite dans le cadre de la Révision générale des politiques publiques, cette réforme a dramatiquement redimensionné l'État départemental, principal point d'accès et partenaire des communes, notamment des plus petites, au profit de l'État régional et du préfet de région. Le ministère de l'Écologie (anciennement de l'Équipement) et ses directions départementales ont particulièrement souffert. Avec l'administration préfectorale, la présence d'un réseau très fin de représentants locaux de l'État sur les territoires était aussi un élément favorable à l'influence des maires. Le cas du ministère de l'Équipement était topique de ce point de vue. La présence d'un réseau de production en régie de services aux collectivités faisait de chaque maire un client potentiel du ministère et entretenait un canal de relation permanent entre le local et l'État. En affaiblissant ce réseau, la REATE n'a pas seulement affaibli l'État local au profit des directions centrales des ministères, des agences et des préfets de région, elle a aussi privé les maires, tout particulièrement ceux représentant les petites communes, de voies d'accès au centre et à l'État en général.

La construction européenne, enfin, a dramatiquement changé la donne. Le développement des politiques européennes, notamment des politiques régionales réformées à partir de 1988, a érigé la Commission européenne en interlocuteur des collectivités. Si l'État, notamment en France, a toujours su conserver une position stratégique dans la définition des politiques régionales ${ }^{22}$, le principe du partenariat a néanmoins fait des collectivités des protagonistes de la fabrique des politiques européennes. C’est d'ailleurs, avec la création du Comité des régions, la réforme en 1988 des principes de programmation des fonds structurels dans le sens d'une implication des régions qui a suscité l'émergence de la théorie de la gouvernance multi-niveaux, faisant de la construction européenne non plus un processus strictement intergouvernemental qui n'implique que les États membres mais une dynamique qui sollicite une diversité de protagonistes (institutions communautaires, collectivités locales, intérêts organisés). Au-delà de la politique régionale, la

21. Philippe Bezes, Patrick Le Lidec, «L'hybridation du modèle territorial français », Revue française d'administration publique, vol. 136, $\mathrm{n}^{\circ}$ 4, 2011, p. 919-942.

22. Ian Bache, «The Extended Gatekeeper: Central Government and the Implementation of EC Regional Policy in the UK», Journal of European Public Policy, vol. 6, n 1, 1999, p. 28-45. 
construction européenne a eu aussi pour effet de créer un vaste espace de transfert de politiques publiques ${ }^{23}$ où s'échangent des solutions, des savoir-faire et des best practices qui ont toujours plus d'impact sur les politiques locales. Mais, bien entendu, toutes les collectivités et tous les maires ne sont pas situés sur un pied d'égalité pour accéder à cette plateforme de transferts et encore moins pour s'y imposer comme trends setters, ou précurseur de tendance.

Cette nouvelle donne modifie significativement les systèmes relationnels dans lesquels sont intégrés les maires. Ces systèmes se sont globalement multilatéralisés. Déjà, dans la foulée de la décentralisation, la contractualisation des politiques publiques avait fait émerger les régions comme intermédiaires dans les relations entre les communes et l'État. La montée en puissance des politiques régionales de l'Union européenne a consolidé les dispositifs multi-acteurs inaugurés par la contractualisation. Les incitations de l'État mais aussi le progressif repli de ses administrations déconcentrées ont donné un coup de pouce à la coopération intercommunale et donc à ces relations horizontales auxquelles les maires s'ingéniaient à échapper dans la configuration précédente. La constitution d'un espace européen de financement des politiques locales mais aussi d'échanges des modèles de politique publique a aussi conduit à un développement de relations horizontales transnationales. Les réseaux de collectivités locales, dont Eurocités est sans doute le plus emblématique, sont devenus des espaces essentiels de construction du sens des politiques locales. Pour reprendre le langage de la théorie de la gouvernance multi-niveaux, les rapports intergouvernementaux ne sont plus nested, autrement dit ils ne sont plus cantonnés à un espace national et à un dialogue bilatéral État-commune.

De manière générale, les ressources pour les politiques publiques ne sont plus principalement aux mains de l'État. Elles doivent être trouvées à d'autres guichets (intercommunalité, régions, Union européenne, associations nationales, transnationales). De surcroît, du fait des réformes de privatisation, de libéralisation et, plus généralement, du fait du basculement graduel des rapports de force entre public et privé au profit du second pôle, les ressources pour l'action publique ne sont plus le monopole des institutions publiques. Ces ressources, financières, cognitives et d'expertise, doivent être mobilisées du côté de la société civile, des intérêts organisés, des forces économiques et des groupes professionnels.

23. Claudio Radaelli, «Policy Transfer in the European Union: Institutional Isomorphism as a Source of Legitimacy», Governance, vol. 13, 1, 2000, p. 25-43. 
Cela est tout particulièrement vrai pour les villes, conduisant les maires à structurer des coalitions durables avec les représentants de ces groupes, ce dont les travaux sur la gouvernance urbaine ou les régimes urbains se sont faits l'écho ${ }^{24}$.

Contrairement au système de relations intergouvernementales précédent, centré sur la relation verticale maire-État, le système qui s'est récemment structuré n'est plus nivelant mais au contraire largement différenciant et potentiellement inégalitaire. Tous les maires ne sont pas affectés de la même manière par l'émergence d'un système relationnel multilatéral et de la gouvernance multi-niveaux. C'est ici que l'inanité de l'approche générique du local apparaît au grand jour et qu'il faut distinguer les situations des maires en fonction des espaces qu'ils gouvernent et des ressources que ces espaces leur fournissent.

108 Le repositionnement de l'État, la transformation des rapports centrepériphérie et la métropolisation font donc des gagnants et des perdants parmi la vaste - et peu utile analytiquement - catégorie des «maires».

Ce qui fait la différence, c'est qu'alors qu'auparavant la simple captation de ressources politiques garanties par la détention de mandats et leur cumul assurait un minimum de capacité d'influence à un grand nombre de maires, aujourd'hui c'est la possibilité de mobiliser des ressources pour l'action publique au niveau du territoire qui active une dynamique vertueuse de «montée en influence». La possibilité qu'a un maire urbain président de communauté urbaine de mobiliser des ressources financières, cognitives et d'expertise sur un territoire dynamique sur le plan économique et démographique, d'y construire des coalitions, lui assurera l'accès à d'autres ressources. La capacité qu'il aura à développer un spectre large de politiques urbaines innovantes attirera les fonctionnaires et techniciens ambitieux. Elle suscitera l'intérêt de l'État qui tend de plus en plus à la fois à attribuer ses ressources aux collectivités dans le cadre d'appels d'offres compétitifs où il récompense non plus les plus nécessiteux mais les plus méritants ${ }^{25}$ et à calquer ses propres programmes

24. Clarence Stone, Regime Politics: Governing Atlanta, 1946-1988, Lawrence (Kan.), University Press of Kansas, 1989; Alan Harding, "Urban Regimes in a Europe of the Cities?", European Urban and Regional Studies, vol. 4, n 4, 1997, p. 291-314; Patrick Le Galès, Le Retour des villes européennes. Sociétés urbaines, mondialisation, gouvernement et gouvernance, Paris, Presses de Sciences Po, 2003; Gilles Pinson, Gouverner la ville par projet. Urbanisme et gouvernance des villes européennes, Paris, Presses de Sciences Po, 2009; le même, «La gouvernance des villes françaises. Du schéma centre-périphérie aux régimes urbains », Metropoles.revues.org, vol. 7, 2010.

25. Renaud Epstein, «Les trophées de la gouvernance urbaine», Pouvoirs locaux, vol. 97, 1997. 
sur les innovations des gouvernements urbains les plus en pointe, ce qui prédispose ces derniers à emporter la mise ${ }^{26}$. L'abondance des ressources, la capacité à innover et le soutien de l'État permettront à ce maire et à ses collaborateurs de se projeter à l'échelle européenne, dans les réseaux de villes notamment, et d'y faire valoir une image d'exemples à suivre. À l'inverse, le maire d'une commune pauvre de périphérie urbaine ou en milieu rural peinera à mobiliser des ressources localement et à faire valoir cette excellence, et ce dénuement en ressources risque d'être de moins en moins compensé par les mannes de l'État. Aujourd'hui, ce type de commune, confronté au redimensionnement drastique de l'administration territoriale de l'État, se trouve privé des ressources redistributives que cette dernière offrait et des points d'accès aux circuits de décision de l'État.

Les maires de ce deuxième type de ville ne vivent définitivement plus dans le même monde qu'un Gérard Collomb, sénateur-maire de Lyon et président de la communauté urbaine dite du Grand Lyon. Ce dernier gouverne une machine technique et administrative, le Grand Lyon, dont le budget équivaut à celui de la région Rhône-Alpes et qui bénéficie de bases fiscales rendues dynamiques par l'expansion économique et démographique de la métropole lyonnaise. Si l'accès au centre reste pour lui stratégique, pour obtenir notamment l'adoption du statut de métropole pour le Grand Lyon, les relations avec les milieux économiques locaux, la chambre de commerce, le système universitaire local, et bien entendu les relations horizontales avec les autres maires de la communauté urbaine le sont tout autant sinon davantage. Les services déconcentrés de l'État sont devenus de plus en plus discrets à l'échelle du Grand Lyon. Même la rénovation urbaine, pourtant symbole du retour de l'État sous les auspices du "gouvernement à distance ${ }^{27}$ », est largement prise en charge par la communauté urbaine. Alors que bon nombre de maires ruraux ont troqué une dépendance vis-à-vis de l'État pour une dépendance moins pourvoyeuse en ressources vis-à-vis du département et parfois de la région, le maire-président du Grand Lyon a absorbé les compétences du département du Rhône sur le territoire de la communauté urbaine et se plaît à concurrencer la région RhôneAlpes sur bon nombre de ses compétences (enseignement supérieur et

26. Vincent Béal, Les Politiques du développement durable. Gouverner l'environnement dans les villes françaises et britanniques (1970-2010), thèse de science politique, Université Jean-Monnet Saint-Étienne, 2011.

27. Renaud Epstein, «Les trophées de la gouvernance urbaine», art. cité. 
recherche, développement économique) ${ }^{28}$. Enfin, le maire et ses suiveurs ont largement investi les réseaux européens à la fois pour y glaner des ressources et y accréditer une image d'excellence et d'innovation propre à attirer à Lyon d'autres ressources.

*

Ainsi le système relationnel vertical et bilatéral mais relativement égalitaire et nivelant typique de la régulation croisée a-t-il laissé la place à un système de gouvernance multi-niveaux où les relations se sont diversifiées et multipliées mais où, moins que jamais, un maire n'en vaut un autre. Le nouveau système relationnel ouvert et multilatéralisé semble fonctionner d'après le principe du winner takes all selon lequel le mieux pourvu en ressources est celui qui peut le mieux profiter des relations intergouvernementales, censées au départ pourtant profiter aux moins pourvus en ressources. On retrouve là une des caractéristiques et des ambiguïtés fondamentales des fonctionnements en réseaux: ils sont à première vue horizontaux, relativement ouverts et pluralistes, mais leurs effets redistributifs peuvent s'avérer finalement préjudiciables aux plus faibles.

Pour autant, a-t-on complètement perdu au change ? Rien n'est moins sûr! Les membres du Centre de sociologie des organisations avaient bien montré comment le système relationnel de la régulation croisée avait engendré clientélisme et passivité chez les élus. Ils évoquaient «le cercle vicieux de la technocratie et de l'irresponsabilité locale», la «diminution progressive parmi les élus locaux, non seulement de toute capacité, mais même de toute volonté de création autonome ${ }^{29}$ ». Les maires étaient plus occupés à une activité de courtage et de captation de ressources externes qu'à une activité de mobilisation de ressources locales et d'organisation des systèmes d'action locaux. Pis, ce système de relation et de dépendance verticales confortait la position exagérément prépondérante des maires, voire leur omnipotence dans les espaces sociopolitiques locaux, jusqu'à étouffer toute capacité de mobilisation et d'auto-organisation. Par ailleurs, on le sait, la régulation croisée a été très favorable aux intérêts des maires des petites communes, notamment

28. Gilles Pinson, Deborah Galimberti, «Métropoles franches et régions agencifiées », Pouvoirs locaux, vol. 96, 2013, p. 48-55.

29. Jean-Pierre Worms, Pierre Grémion, «Le préfet et les collectivités territoriales », Esprit, janvier 1970. 
rurales, et a empêché l'émergence à l'agenda des enjeux proprement urbains.

Aujourd'hui, le fait urbain et métropolitain est enfin reconnu. Les maires ont pris la mesure de leur responsabilité en matière de mobilisation locale autour d'un projet. Ils ont d'une certaine manière préparé leur territoire à un retrait progressif de l'État. Reste à régler la question des rapports entre, d'une part, les territoires les mieux organisés, les mieux dotés en ressources et quasiment affranchis de la dépendance à l'État (les plus prospères des métropoles) et, d'autre part, ceux qui peinent à mobiliser ces ressources et à s'organiser face au démantèlement de l'État local.

Les maires ont pu constituer jadis un groupe homogène, uni par le même type de relations bilatérales et verticales avec un partenaire dominant, l'État. Ces relations étaient typiques des rapports centre-périphérie asymétriques qui prévalaient dans les systèmes dits "napoléoniens» de gouvernement local. Pour les maires, elles se traduisaient par une subordination juridique au centre mais aussi par une grande capacité d'accès à ce même centre, via notamment le cumul des mandats. Ce système relationnel a volé en éclats au profit d'un autre, davantage multilatéral et dans lequel l'État a perdu le monopole des relations avec les maires. Ce système relève de ce que les spécialistes de l'Union européenne ont appelé "la gouvernance multi-niveaux». Il est beaucoup plus ouvert, pluraliste, mais il est en même temps gros de logiques de différenciation et d'inégalités dans l'accès aux ressources. Les grandes métropoles et leurs maires-présidents sortent grands gagnants de ce système en voie d'émergence. 